\title{
Enhanced Channel Estimation and Performance Analysis Using H-Infinity Filter for MIMO-Orthogonal Frequency Division Multiplexing Systems
}

\author{
Joseph Gladwin Sekar and Salivahanan Sankarappan \\ Department of ECE, SSN College of Engineering, Kalavakkam, India
}

\section{Article history}

Received: 15-05-2014

Revised: 29-05-2014

Accepted: 12-09-2014

Corresponding Author:

Joseph Gladwin Sekar

Department of ECE, SSN College of Engineering, Kalavakkam, India Email: josephs@ssn.edu.in

\begin{abstract}
This paper, deals with H-infinity channel estimation technique for Orthogonal Frequency Division Multiplexing (OFDM) systems, in fading channel. Channel estimation is important for wireless applications because, in practical transmission scenario, channel correlation functions are either not known or cannot be easily estimated. It is therefore desirable to have an estimator which is robust to mismatches between the assumed and the actual channel correlation functions. The wireless channel has fading characteristics and its time varying nature adds additional cost to estimator design. A simplified Kalman filter is proposed which reduces the noise effects of the least square estimation. While the Kalman filtering algorithm requires the noise process to be zero mean besides the requirement to know the standard deviation of the noise process, H-infinity filtering algorithm makes no assumption about the noise and hence is said to be more robust in the wake of uncertainty. The proposed channel estimation algorithm is more robust in terms of model uncertainty and is more suitable for OFDM systems. Further, the complexity of the H-infinity filter is not too high. Given the conditions, H-infinity poses a suitable filter than any other, for channel estimation.
\end{abstract}

Keywords: OFDM, MIMO, Channel Estimation, Channel, H-Infinity

\section{Introduction}

Wireless communication has become indispensable as it envisages the need of high data rate capable technologies. An inherent trait of wireless devices is mobility which unfortunately the wired counterparts failed to offer. The reduction in cost for installation and maintenance further amplifies the dire need of wireless communication. However a major setback of this type of communication is the noisy environment which most of wired equipments might have escaped from. Added to the problem of noise, wireless communication must as well combat the problems of fading that arises due to multipath propagation. Nevertheless wireless communication has always established supremacy over wired communication.

Though the aforesaid problems cannot be completely eliminated, yet their effects can certainly be reduced or compensated more enough, so that the effect is made less harmful. Channel estimation comes handy for this sort of a problem. Though a lot of channel estimation algorithms have been proposed, of special interest are two algorithms- Kalman and H-infinity.

In this study the two algorithms are compared and analyzed based on their performance. These algorithms are applied to a MIMO-OFDM environment. OFDM systems offer high data rate because of their modulating cum multiplexing ability. They are suitable for transmission over time-variant frequency selective channels (Hijazi et al., 2010). OFDM is one of the multi carrier modulation techniques that transmit signals through multiple carriers. These sub-carriers have different frequencies and they are orthogonal to each other. These subcarriers can be considered frequency-flat approximately as the number of the sub-channel $N$ is large enough. Hence the OFDM signals will suffer channel distortion less than the conventional 
modulated signals. Combining this technique with MIMO technology has attracted attention in wireless communications; because it offers significant increase in data throughput and link range without additional bandwidth or increased transmit power.

The baseband data considered is a $32 \times 32$ grey scale image replicated 8 copies. Each of these 8 copies is transmitted on a sub-carrier and undergoes fading as it is passed through the channel. In addition, the AWGN noise is also added to each of these images. The reason to have replicated images is that it represents multipath propagation in a frequency selective fading environment, where each path is assumed to have uncorrelated fading. Therefore even if the data in a certain sub-carrier is lost, it can always be retrieved by the other sub-carriers. The probability of data loss in every sub-carrier is thus made very less. By estimation we choose the best sub-carrier that is least deteriorated by fading and noise effects so that its error value becomes certainly less while other subcarriers may still pose a large error value.

Finally the efficiency of the algorithms is validated and the amount of the original information retrieved is compared in both the cases.

The study is organized as follows. Section II describes the channel model and the system equations. Section III describes the two estimation algorithms. Finally section IV gives the performance comparison of the algorithms. Conclusion and discussion are subsequently.

\section{System Model}

Consider a MIMO-OFDM system with $K$ subcarriers, $N_{t}$ transmit antennas and $N_{r}$ receive antennas. Further, the input to the system is a grey scale image. The image is replicated into $K$ copies and each of them is transmitted through a sub-carrier. The channel is modelled as a Rayleigh fading channel. The images are transmitted through this channel where they are corrupted by additive white Gaussian noise. The channel model considered in this work is represented by Fig. 1 .

Further the system is described as a linear dynamic system. The system equations are as follows Equation 1 and 2 :

$$
\begin{aligned}
& X_{k+1}=A X_{k}+B U_{k}+W_{k} \\
& Y_{k}=C X_{k}+V_{k}
\end{aligned}
$$

where, $A, B, C$ are known matrices, $k$ is time index, $X$ represents the state of the system, $U$ and $Y$ are input to the system and measured output respectively and $W, V$ represents noise.

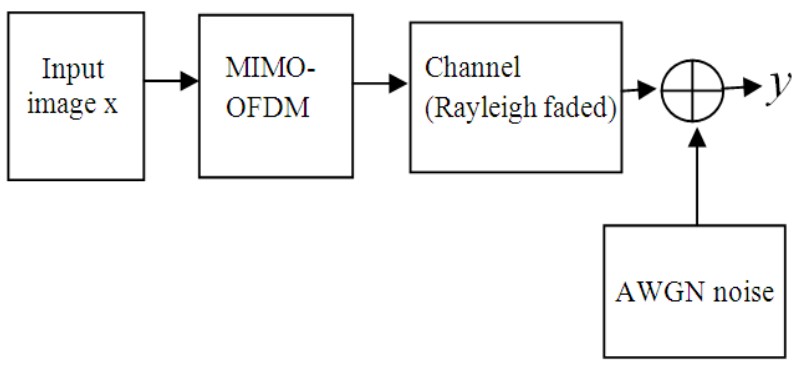

Fig. 1. Channel model

\section{Estimation Algorithms}

\section{Kalman Filtering Algorithm}

The algorithm works in a two-step process. In the prediction step, the Kalman filter produces estimates of the current state variables, along with their uncertainties. Once the outcome of the next measurement (necessarily corrupted with some amount of error, including random noise) is observed, these estimates are updated using a weighted average, with more weight being given to estimates with higher certainty. Because of the algorithm's recursive nature, it can run in real time using only the present input measurements and the previously calculated state; no additional past information is required (Nasseri and Bakhshi, 2010). From a theoretical standpoint, the main assumption of the Kalman filter is that the underlying system is a linear dynamical system and that all error terms and measurements have a Gaussian distribution (Schafhuber et al., 2003; Suresh et al., 2012).

Assuming that both exciting term $\mathrm{W}_{k}$ and observation noise $V_{k}$ (additive noise) are white Gaussian processes with zero mean and uncorrelated variances $Q$ and $R$ Equation 3 to 5 :

$E\left\{W_{k} W_{k}^{T}\right\}=Q$

$E V_{k} V_{k}^{T}=R$

And:

$E\left\{W_{k} V_{j}^{T}\right\}=0$

(where, $E$ denotes expectation), the design objective of Kalman filter is to determine the optimal estimate based on the criterion such that Equation 6:

$P_{k}=E\left\{e_{k} e_{k}^{T}\right\}$ 
Is minimum. For the Linear model, the Kalman filtering algorithm is given by the state Equations (i) measurement update Equations 7 to 9:

$$
\begin{aligned}
& \hat{H}_{k}=A \hat{H}_{k+1}+K_{k}\left[Y_{k}-V A \hat{H}_{k-1}\right] \\
& P_{k}=\left[1-K_{k} V^{\prime}\right] P_{k \mid k-1} \\
& K_{k}=P_{k \mid k-1}(V) /\left[R+V P_{k \mid k-1} V^{\prime}\right]
\end{aligned}
$$

(ii) Time update Equation 10:

$$
P_{k \mid k-1}=A P_{k} A^{\prime}+Q
$$

Finally the estimate of the original data can be obtained as Equation 11 and 12:

$$
\begin{aligned}
& \hat{H}_{k}=\hat{H}_{k-1} \\
& X_{k}=Y_{k} * \hat{H}_{k}
\end{aligned}
$$

Where:

$H_{k}=$ Channel estimate at instant $k$

$Y_{k}=$ Corrupted data at instant $k$

$X_{k}:=$ Data estimate at instant $k$

$P_{k}=$ Posteriori error covariance

$P_{k / k-1}=$ Error covariance

$V=$ Original data vector

$K_{k}=$ Kalman gain

$A=$ Scalar value

The error criterion used for Kalman filter is based on the minimum mean square error criterion (Luo and Huang, 2008).

\section{H-Infinity Filtering Algorithm}

One of the reasons for the prominence of the Kalman filter is that it results in the smallest possible standard deviation of the estimation error. In other words, the Kalman filter is the minimum variance estimator. However Kalman filters' assumptions are always not satisfied (Zamiri-Jafarian and Pasupathy, 2007). For instance, the noise may not always be zero mean. Similarly the information on noise statistics may not be available. In such cases a better option would be to use the $\mathrm{H}_{\infty}$ filter which absolutely requires no knowledge of the noise statistics and makes no assumption about the noise (Naganjaneyulu and Satya Prasad, 2007; Peng et al., 2011). For the same linear dynamic system as described by Equations 1 and 2 the following criterion needs to be satisfied Equation 13:

$$
\min \hat{x} \max w, V J
$$

where, $\hat{x}$ is the estimate and $J$ is the measure of how good the estimator is.

$w$ and $V$ are the noise terms that worsen the estimate.

The function $J$ is defined as follows Equation 14:

$J=\frac{a v e\left\|x_{i} x_{k}\right\| q}{\text { ave }\left\|w_{k}\right\|+a v e\left\|V_{k}\right\|}$

The aim will be to find an estimate that makes $J$ small even while the noise terms try to make $J$ large. To this seemingly difficult problem is a solvable solution that states Equation 17:

$$
J<1 / \gamma
$$

where, $\gamma$ is some constant number. That is, an estimate can be found so that the maximum value of $J$ is always less regardless of the values of the noise terms $w$ and $v$. The state equations that forces $J<1 / \gamma$ is given as follows Equation 18 to 21 :

$$
L_{k}=(I)
$$

$L_{k}=\left(I-\gamma Q P_{k}+Y^{T} V^{-1} Y P_{k}\right)^{-1}$

$K_{k}=A P_{k} L_{k} Y^{T} V^{-1}$

$\hat{h}_{k+1}=a \hat{h}_{k}+B u_{k}+K_{k}\left(a-y \hat{h}_{k}\right)$

$P_{(k+1)}=A P_{k} L_{k} A^{T}+W$

Where:

$I \quad=$ Identity matrix

$\gamma \quad=$ Gamma value

$W \quad=$ Process noise

$Q \quad=$ Process noise covariance $\left(E\left(W W^{T}\right)\right)$

$V=$ measurement noise

$P \quad=$ Error covariance matrix

$\hat{h}=$ Channel estimate

$a \quad=$ Original data 


$$
\begin{array}{ll}
y & =\text { Corrupted data } \\
K & =\text { Gain } \\
A, B & =\text { Known matrices } \\
k & =\text { Time instant }
\end{array}
$$

The equations have the same form as the Kalman filter equations, but the details are different.

One way to guarantee almost zero estimation error is to set $\gamma$ to a very large number, since $1 / \gamma$ will be almost zero. However this cannot be done as, the mathematical derivation of the $H_{\infty}$ equations is valid only if $\gamma$ is chosen such that all of the Eigen values of the $P$ matrix have magnitudes less than one. If too large values of $\gamma$ are being chosen, a solution to the $H_{\infty}$ filtering problem does not exist. That is, an estimator cannot be found that will make the estimation error arbitrarily small.

\section{Simulation Results}

We simulate the convergence property and the error performance of the proposed Kalman and $H_{\infty}$ estimators. The system parameters are organized as follows: MIMOOFDM systems under AWGN, Rayleigh multi-path fading channel, QAM modulated OFDM symbols.

Primarily a $32 \times 32$ grayscale image is taken and OFDM modulation is applied to it. The pilots are inserted periodically in the data and a copy of which is marked to the receiver. This is then replicated as 8 copies with $\mathrm{K}=8$. Subsequently each of these is transmitted through a channel modeled as a Rayleigh fading one. AWGN noise is added and the corrupted data with pilots are demodulated. Finally pilot estimation is performed for all the 8 sets simultaneously. The state equations of both the $H_{\infty}$ and the Kalman filter are applied to the corrupted pilots to find a channel estimate. Estimation process is iterated as long as at least one of the sub-carriers poses the least error value. The corresponding subcarrier is adjudged to have undergone minimal fading.

The data and the parameters chosen for both the filters are same. The state equations are iterated until a reasonably close estimate of the channel is obtained. Under these conditions the performance of the two filters is compared under two aspects:

- No of iterations to obtain a reasonably close channel estimate

- The final error obtained between the data and the estimated data

The simulation environment is described in Table 1.

It is observed that $H_{\infty}$ filter outperforms the Kalman filter in both the cases. The following Table 2 elucidates the above.

The following graph shown in Fig. 2 is the plot of error Vs. the no of iterations with respect to pilot symbol estimation. As the number of iterations increases the error decreases. It is interesting to note that the smallest error in case of Kalman filter however does not appear to be the final convergent error.

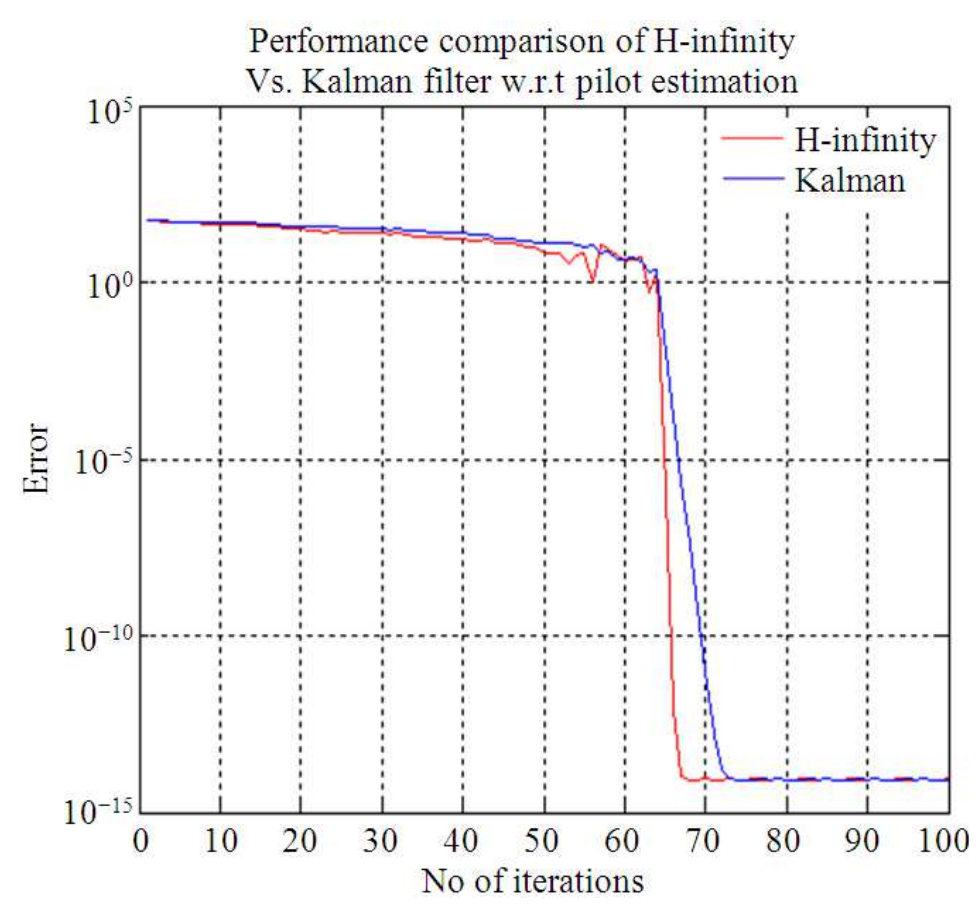

Fig. 2. Performance comparison graph 


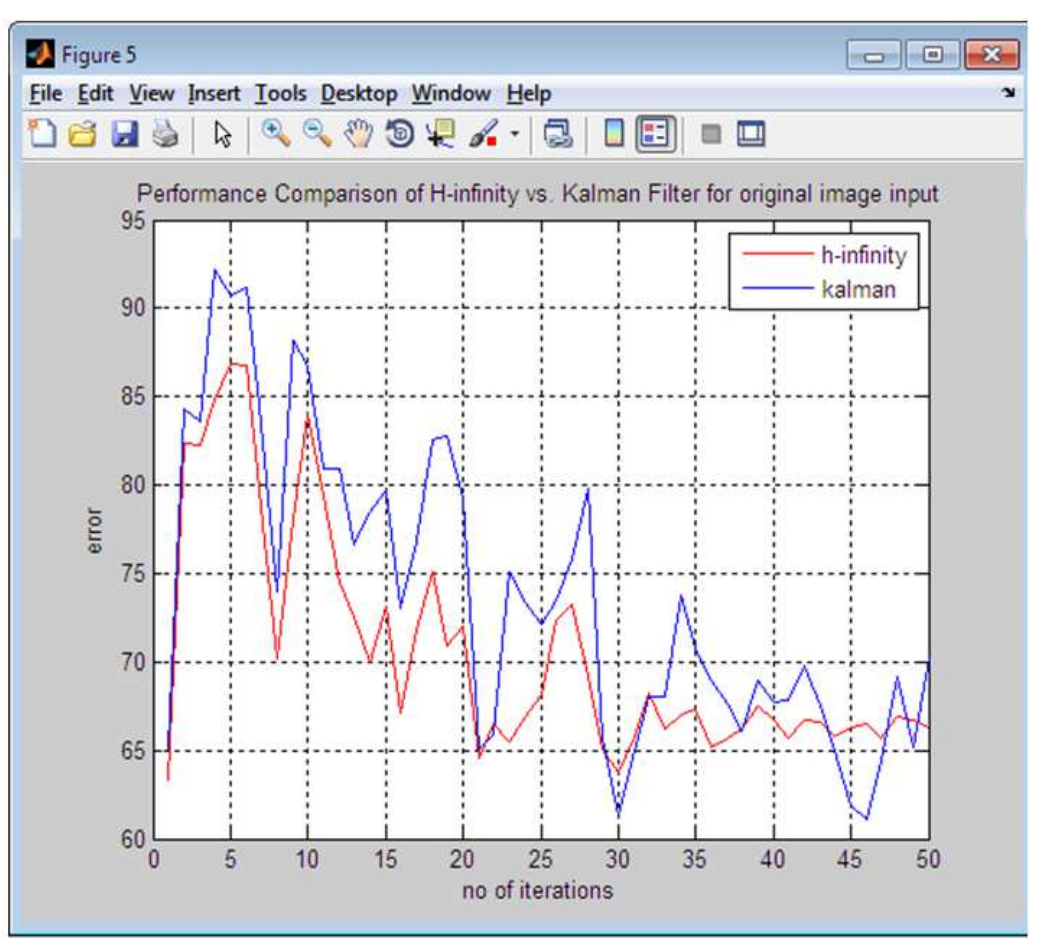

Fig. 3. Performance comparison graph for image input

Table 1. Simulation environment

\begin{tabular}{ll}
\hline Parameter & Value \\
\hline $\mathrm{Nt}$ & 8 \\
$\mathrm{Nr}$ & 8 \\
$\mathrm{~K}$ & 8 \\
Image size & $32 \times 32$ \\
Pilot size & 256 \\
$\mathrm{~W}$ & 0.0003 \\
$\mathrm{~V}$ & 0.01 \\
$\mathrm{Q}$ & 0.01 \\
$\gamma$ & 0.01 \\
\hline
\end{tabular}

Table 2. Performance comparison between $H_{\infty}$ and Kalman filters

\begin{tabular}{lll}
\hline Type of filter & Error value & No. of iterations \\
\hline Kalman & $1.8319 \mathrm{e}-015$ & 74 \\
$\mathrm{H}_{\infty}$ & $1.2212 \mathrm{e}-015$ & 67 \\
\hline
\end{tabular}

Table 3. Performance comparison between $H_{\infty}$ and Kalman filters

\begin{tabular}{lll}
\hline Type of filter & Error value & No. of iterations \\
\hline Kalman & 0.0682 & 50 \\
$H_{\infty}$ & 0.0666 & 50 \\
\hline
\end{tabular}

After estimating the pilots, the image obtained by pilot estimation is compared with the original image to find the error in case of $H_{\infty}$ and Kalman estimators. In the present case, however, the no of iterations were bound to 50 in both cases. Nevertheless $H_{\infty}$ still establishes superiority over Kalman filter in terms of final convergent error value. Table 3 establishes the below.
The estimated pixels retrieved after pilot estimation is compared with those of the original Image and the error is shown in Fig. 3. The pixels corresponding to $H_{\infty}$ filter more closely resemble the original pixels as compared to Kalman filter.

\section{Conclusion and Future Work}

Novel techniques such as OFDM and MIMO stand as promising choices for present high data-rate systems. The Kalman estimator can obtain the optimal performance; however, the complexity is high because of the use of the noise statistics. So, the H-infinity channel Estimator which has remarkably good performance with much less complexity and more robustness than the Kalman counterpart is being designed for MIMO with OFDM systems. H-infinity estimator outperforms Kalman estimator remarkably and has good convergence lesser number of iterations. The effectiveness of the method has been demonstrated based on measurement data and computer simulations. Since the design criterion is based on the worst case disturbance, the $H_{\infty}$ filtering approach is less sensitive to uncertainty in the exogenous signal statistics and system model dynamics.

The proposed $H_{\infty}$ estimator can be applied to next generation wireless protocol so that the fidelity of the system can be improved further. 


\section{Acknowledgment}

The researchers thank the host institution for providing the necessary infrastructure and facilities to carry out the research.

\section{Author's Contributions}

All authors equally contributed in this work.

\section{Ethics}

This article is original and contains unpublished material. The corresponding author confirms that all of the other authors have read and approved the manuscript and no ethical issues involved.

\section{References}

Hijazi, H., E.P. Simon, M. Lienard and L. Ros, 2010. Channel estimation for MIMO-OFDM systems in fast time-varying environments. Proceedings of the 4th International Symposium on Communications, Control and Signal Processing, Mar. 3-5, IEEE Xplore Press, Limassol, pp: 1-6. DOI: $10.1109 /$ ISCCSP.2010.5463392

Luo, Z. and D. Huang, 2008. General MMSE channel estimation for MIMO-OFDM systems. Proceedings of the IEEE 68th Conference on Vehicular Technology VTC 2008-Fall, Sep. 2124, IEEE Xplore Press, Calgary, BC, pp: 1-5. DOI: 10.1109/VETECF.2008.151

Naganjaneyulu, P.V. and K. Satya Prasad, 2007. An adaptive blind channel estimation for OFDM systems tith multimedia by enhanced H-infinity approach. Proceedings of the International Conference on Computational Intelligence and Multimedia Applications, Dec. 13-15, IEEE Xplore Press, Sivakasi, Tamil Nadu, pp: 252-261. DOI: 10.1109/ICCIMA.2007.376
Nasseri, M. and H. Bakhshi, 2010. Iterative channel estimation algorithm in multiple input multiple output orthogonal frequency division multiplexing systems.

J. Comput. Sci., 6: 224-228. DOI: $10.3844 /$ jcssp.2010.224.228

Peng, X., J. Wang and F. Xin, 2011. Improved EMbased H-infinity channel estimator for MIMOOFDM systems. Proceedings of the 7th International Conference on, Wireless Communications, Networking and Mobile Computing, Sept. 23-25, IEEE Xplore Press, Wuhan, pp: 1-4. DOI: 10.1109/wicom.2011.6036642

Schafhuber, D., G. Matz, F. Hlawatsch, 2003. Kalman tracking of time-varying channels in wireless MIMO-OFDM systems. Proceedings of the Conference Record of the 37th Asiloma Conference on Signals, Systems and Computers, Nov. 9-12, IEEE Xplore Press, pp: 1261-1265. DOI: 10.1109/ACSSC.2003.1292191

Suresh, M.N., S.J. Thiruvengadam and V. Abhaikumar, 2012. Symbol timing estimation in multiband orthogonal frequency division multiplexing based ultrawide band system. Am. J. Applied Sci., 9: 505-509. DOI: 10.3844/ajassp.2012.505.509

Zamiri-Jafarian, H. and S. Pasupathy, 2007. Robust and improved channel estimation algorithm for MIMOOFDM systems. IEEE Trans. Wireless Commun., 6: 2106-2113. DOI: 10.1109/TWC.2007.05718 